\title{
Holoprosencephaly-postaxial polydactyly syndrome
}

INSERM

\section{Source}

INSERM. (1999). Orphanet: an online rare disease and orphan drug data base.

Holoprosencephaly-postaxial polydactyly syndrome. ORPHA:2166

Holoprosencephaly-postaxial polydactyly syndrome associates, in chromosomally

normal neonates, holoprosencephaly, severe facial dysmorphism, postaxial polydactyly and other congenital abnormalities, suggestive of trisomy 13 (see this term). 